\title{
Extra pulmonary tuberculosis: A retrospective study in Matara District, Sri Lanka
}

\author{
Mohotti BK ${ }^{1}$, Wadanamby DCW 2 , WanigapuraHR ${ }^{3}$, MadhaveeTHC $^{3}$, \\ Mallikarachchi $\mathrm{KH}^{3}$
}

\begin{abstract}
Background: Despite improvements in prevention, diagnosis and treatment, tuberculosis continues to be a leading cause of death globally. Since extra pulmonary tuberculosis does not contribute significantly to the transmission of the disease, it is not given priority in public health agenda. But it contributes significantly to tuberculosis related morbidity and mortality.

Objectives: To identify the various presentations of extra pulmonary tuberculosis in Matara district, Sri Lanka and to evaluate the response to anti tuberculosis treatment.

Methods: A descriptive study was conducted among the patients registered in District Chest Clinic, Matara. Data analysis were done by using SPSS version 25 . Chi-square test was used to assess the association between variables.

Results: Among 147 patients 50.3\% were female (mean age = 44years). 93.2\% were Sinhalese. $11.6 \%$ of the study population were from Matara municipal council $\mathrm{MOH}$ area. $32.7 \%$ of the sample was diagnosed to have pleural effusion. 9.5\% were current smokers. 9.5\% were diagnosed with diabetes.98\% of patients had completed treatment.

Conclusions: There was high prevalence of pleural tuberculosis. The diagnosis of lymphatic tuberculosis was lower. Prevalence of extra pulmonary tuberculosis was high in $\mathrm{MOH}$ areas with high population density. Significantly high treatment completion rate than the national target was noted at district chest clinic Matara.
\end{abstract}

Introduction

Tuberculosis is an infectious disease, which is air born and caused by the bacillus Mycobacterium tuberculosis. (Occasionally by Mycobacterium bovis and Mycobacterium africanum). ${ }^{1}$ Tuberculosis can involve any part of the human body other than nails, teeth and hair, with lung being the most common. Extra pulmonary tuberculosis refers as any bacteriologically confirmed or clinically diagnosed case of tuberculosis involving organs other than lung parenchyma or tracheobronchial tree. ${ }^{2}$ Diagnosis of extra pulmonary tuberculosis should be based on smear/culture positive specimen or histological or strong clinical evidence consistent with active extra pulmonary tuberculosis, followed

1. Consultant physician, District General Hospital, Matara

2. Medical Officer, District Chest Clinic, Matara.

3. Medical Officer, District General Hospital,Matara

\section{Corresponding Author:}

Buddhi Krishanthi Mohotti, District General Hospital, Matara ,Sri Lanka

Email: buddhimohotti@yahoo com

ID https://orcid.org/0000 00023837 613X by a decision by a clinician to treat with a full cause of anti-tuberculosis chemotherapy.1A patient with pulmonary and co-existing extra pulmonary tuberculosis should be classified as pulmonary tuberculosis. $^{2}$

Presentation of extra pulmonary tuberculosis may be atypical and symptoms may depend on the affected anatomical site.Usual anatomical sites of extra pulmonary tuberculosis arebones and joints, central nervous system,genitourinary system, tuberculous lymphadenopathy, abdominal tuberculosis,ocular tuberculosis, ttuberculosis pleural effusion, miliary tuberculosis, pericardial tuberculosis, tuberculosis involving any other organs. $^{1}$

Presentation of extra pulmonary tuberculosis may be atypical or relatively insidious and tuberculosis may not be considered initially in differential diagnosis. This is an important phenomenon as delay in diagnosis may be crippling or even life threatening. Therefore, from a public health perspective, there is a need to address this group of patients as do they contribute to the total burden of the disease and they do have a significant impact on resources. 
Diagnosis of pulmonary tuberculosis is delayed in Sri Lanka. Diagnosis of extra pulmonary tuberculosis is further delayed due to the atypical presentations and due to the difficulty in the process of diagnosis. Delay in diagnosis of extra pulmonary tuberculosis results in high morbidity and mortality.

Considering the burden of extra pulmonary tuberculosis in Sri Lanka it is important to study this group of patients. Descriptive studies on extra pulmonary tuberculosis in Sri Lanka are inadequate. This study may provide the basis of further studies and might help in planning and policy making in the future and also to achieve the end TB strategy in 2025.

Annual case detection of tuberculosis in Sri Lanka approximates to ten thousand cases, while extra pulmonary tuberculosis cases make up to about two thousand five hundred among them(25\%).1When considering Matara district, in 2017 there were72 extra pulmonary tuberculosis cases out of 225 (32\%) total and, In 2018, 81 cases registered out of total $216(37.5 \%) .^{3}$

Matara district is located in south west of Sri Lanka and has an area of 1,282.5 square kilometers and has a population of 814,048 in 2012 . Population density in Matara is 635 per square kilometer. The female population is (422.145) somewhat higher than the male population $(389,903) .{ }^{4}$ There are seventeen $\mathrm{MOH}$ areas in Matara district.

The Objectives of this study were,

1. To determine socio-demographic features of extra pulmonary tuberculosis patients in Matara district (Age, Gender, and Race)

2. To describe the anatomical site involved in of extra pulmonary tuberculosis. (As mentioned in the introduction)

3. To describe the prevalence of extra pulmonary tuberculosis by $\mathrm{MOH}$ areas.

4. To describe association with co-morbidities ( HIV, diabetes mellitus, rheumatoid arthritis, chronic kidney disease, malignancy, chronic respiratory disease) and correlation with smoking, alcohol and substances use.

5. To describe the treatment outcome (Cured, Treatment completed, Loss to follow up, Treatment failure, Died)

\section{Methodology}

This was a descriptive study involving a retrospective review of extra pulmonary tuberculosis registers and extra pulmonary tuberculosis treatment cards. We studied the patients who were registered and treated as extra pulmonary tuberculosis in District Chest Clinic Matara. The study period was 2 years
from1stJanuary 2017 to 31stDecember 2018.

District Chest Clinic. Matara is under technical guidance by the National Programme for Tuberculosis Control and Chest Diseases (NPTCCD) and gives service toall pulmonarydiseases andtuberculosis patients in Matara district.

The study included all cases of extra pulmonary tuberculosis of all age groups and both sexes treated at District Chest Clinic Matara.

Patients who were diagnosed as extra pulmonary tuberculosis and commenced treatment at other district chest clinics and transferred to complete treatment at District Chest Clinic Matara and ppatients who died of tuberculosis or its complications while on treatment irrespective of the period of treatment given were included in this study.

Patients who were not residents of the Matara district, but any how diagnosed at this clinic, were transferred out to the relevant district chest clinic of their own resident area to complete anti tuberculosis chemotherapy (transferred out), patients who were initially diagnosed as extra pulmonary tuberculosis but became sputum positive for Acid fast Bacilli culture/Gene expert (a molecular diagnosis technique which identifies the DNA molecule of the Mycobacterium tuberculosis)or for both during the period of treatment (Any case of pulmonary tuberculosis) and patients who died not due to tuberculosis or its complications while on treatment irrespective of the period of treatment,were excluded from the study.

The ethical clearance was obtained from the ethical review committee, University of Ruhuna. The study sample was 147 (70 patients from 2017 and 77 patients from 2018). Data collection was done using SPSS data sheet. Data was collected from patient's record according to the tuberculosis registration number which was given by District Chest Clinic, Matara.

Missing data in the records were traced over the phone as patient's records mentioned their active telephone numbers. There was no need for visiting patients or getting the help of public health inspectors.

Before gathering missing data from patients over the phone, informed consent was obtained verbally. Data was analysed using SPSS statistics 25 . Chi-square test was used to assess the association between variables. 


\section{Results}

The study group contained total number of 147 patients who were registered as extra pulmonary tuberculosis in District Chest Clinic, Matara.Table 01 showed the distribution of sociodemographic data among study population. The mean age was 44 years and standard deviation was $18.006 .50 .3 \%$ were female and $93.2 \%$ were Sinhalese.

Table 01 - Socio-demographic data of study population

\begin{tabular}{|c|c|c|}
\hline Category & Frequency & Percentage \\
\hline \multicolumn{3}{|l|}{ Gender } \\
\hline Male & 73 & 49.7 \\
\hline Female & 74 & 50.3 \\
\hline \multicolumn{3}{|l|}{ Ethnic group } \\
\hline Sinhalese & 137 & 93.2 \\
\hline Sri Lankan Moor & 7 & 4.8 \\
\hline Tamil & 3 & 2.0 \\
\hline \multicolumn{3}{|l|}{ Age } \\
\hline $1-10$ & 2 & 1.3 \\
\hline $11-20$ & 9 & 6.1 \\
\hline $21-30$ & 25 & 17.0 \\
\hline $31-40$ & 32 & 21.8 \\
\hline $41-50$ & 19 & 12.9 \\
\hline $51-60$ & 29 & 19.7 \\
\hline $61-70$ & 18 & 12.2 \\
\hline $71-80$ & 9 & 6.1 \\
\hline $81-90$ & 4 & 2.7 \\
\hline
\end{tabular}

8 patients out of 147 (5.4\%) had got contacted with a tuberculosis patient and 2 patients (1.4\%) gave a history of past tuberculosis infection.

As mentioned in table 02 majority of the sample consisted of patients with pleural effusion (32.7\%) followed by lymphatic systeminvolvement (23.8\%).

Table 02- Distribution of anatomical site involved in extra pulmonary tuberculosis

\begin{tabular}{|l|l|l|}
\hline Category & Frequency & Percentage \\
\hline bones and joints & 10 & 6.8 \\
\hline central nervous system & 13 & 8.8 \\
\hline genito urinary tract & 5 & 3.4 \\
\hline lymphatic system & 35 & 23.8 \\
\hline Abdomen & 10 & 6.8 \\
\hline Ocular & 15 & 10.2 \\
\hline pleural effusion & 48 & 32.7 \\
\hline Cutaneous & 4 & 2.7 \\
\hline Other & 7 & 4.8 \\
\hline Total & 147 & 100.0 \\
\hline
\end{tabular}

When considered the $\mathrm{MOH}$ area wise distribution, $11.6 \%$ cases were in Matara Municipal Counsil area followed by Weligama (10.9\%). Lowest number of cases were found in Kirinda $\mathrm{MOH}$ division. (Table 03)

Table 03-Distribution among $\mathrm{MOH}$ areas

\begin{tabular}{|l|l|l|}
\hline Category & Frequency & Percentage \\
\hline Akuressa & 11 & 7.5 \\
\hline Athuraliya & 6 & 4.1 \\
\hline Devinuwara & 9 & 6.1 \\
\hline Dickwella & 8 & 5.4 \\
\hline Hakmana & 13 & 8.8 \\
\hline Kamburupitiya & 13 & 8.8 \\
\hline Kirinda & 2 & 1.4 \\
\hline Kotapola & 4 & 2.7 \\
\hline Matara MC & 17 & 11.6 \\
\hline Kekanadura & 4 & 2.7 \\
\hline Malimbada & 13 & 8.8 \\
\hline Morawaka & 4 & 2.7 \\
\hline Mulatiyana & 4 & 2.7 \\
\hline Pasgoda & 7 & 4.8 \\
\hline Thihagoda & 7 & 4.8 \\
\hline Weligama & 16 & 10.9 \\
\hline Welipitiya & 9 & 6.1 \\
\hline Total & 147 & 100.0 \\
\hline
\end{tabular}

Prevalence of co-morbidities among the study population was distributed as in table 04.

Table 04: Distribution of co-morbidities

\begin{tabular}{|l|l|l|}
\hline Category & Frequency & Percentage \\
\hline CKD & 4 & 2.7 \\
\hline Diabetes & 14 & 9.5 \\
\hline HIV & 1 & 0.7 \\
\hline Malignancy & 2 & 1.4 \\
\hline Rheumatoid Arthritis & 0 & 0 \\
\hline $\begin{array}{l}\text { Chronic Respiratory } \\
\text { Diseases }\end{array}$ & 3 & 2.1 \\
\hline
\end{tabular}

Table 05: Distribution of risk factors

\begin{tabular}{|l|c|c|}
\hline Category & Frequency & Percentage \\
\hline Smoking currently & 14 & 9.5 \\
\hline smoking previously & 16 & 10.9 \\
\hline smoking none & 117 & 79.6 \\
\hline Alcohol currently & 21 & 14.9 \\
\hline Alcohol previously & 16 & 10.9 \\
\hline Alcohol none & 110 & 74.8 \\
\hline $\begin{array}{l}\text { substance abuse } \\
\text { none }\end{array}$ & 147 & 100 \\
\hline
\end{tabular}


According to table 05, 9.5\% cases were current smokers while $10.9 \%$ had smoked previously. Majority werenonesmokers (79.6\%).14.9\% of them were consuming alcohol currently while $10.9 \%$ had consumed alcohol previously. $74.8 \%$ had not consumed alcohol. There were no substance (cannabis and heroine) abuse cases among the study groupamong both male and female

As shown in the table $06,97.9 \%$ of patients had completed treatment while $0.7 \%$ treatment failures and $1.4 \%$ had died due to tuberculosis while on treatment.

Table 06: Distribution of treatment outcome and Distribution of treatment response

\begin{tabular}{|c|c|c|}
\hline Category & Frequency & Percentage \\
\hline \multicolumn{3}{|l|}{ Treatment outcome } \\
\hline Treatment Completed & 144 & 97.9 \\
\hline Treatment failure & 1 & 0.7 \\
\hline Dead & 2 & 1.4 \\
\hline
\end{tabular}

Correlations between co-morbidities and treatment outcome was cross tabulated and there was no significant association was found. Correlations between risk factors and treatment outcome was notfound in the study population.

\section{Discussion}

In Matara district, in the year 2017 there were 72 (32\%)extra pulmonary tuberculosis cases registered out of 225 (total number of registered TB patients) and, In 2018, 81 cases registered out of total 216 (37.5\%).3 147 extra pulmonary patients were included and analysed in this study which is $33.3 \%$ out of total tuberculosis cases and it is compatible with the national figures (25\%-35\%). ${ }^{1}$

The mean age was 44 and standard deviation was 18.006. $95 \%$ of cases are in between the age 8 to 80 (mean +_2SD). This is compatible with national figures which showed highest burden of the disease is in the most economically productive age group of our society (15-54 years). ${ }^{2}$

There was no gender predominance offemale (50.3\%) as compared to male $(49.7 \%)$. When considered the ethnic groups, $93.2 \%$ of cases were Sinhalese, $4.8 \%$ cases were Sri Lankan Moor while $2 \%$ were Tamil which is compatible with Matara district census-20124( Sinhalese 94.16\%, Sri Lankan moor $2.94 \%$, Tamil $2.86 \%$ ). 8 patients (5.4\%) had got con- tact history of tuberculosis in the past and 2 patients (1.4\%) had got past history of tuberculosis.

When considered the distribution of anatomical sites, most common was pleural effusion (32.7\%), followed by lymphatic system (23.8\%). This distribution is not compatible with national figures where the commonest form is lymphatic system which is $35 \%$ followed by pleural effusions which is $20 \% .1$ This revealed increased diagnosis of pleural tuberculosis by using advanced procedures such as thoracoscopy in the District Respiratory Unit.

The occurrence of tuberculosis in any other organ has shown significant variability in Sri Lanka as well as globally.1This study revealed third common site as ocular tuberculosis (10.2\%) followed by central nervous system (8.8\%), bones and joints $(6.8 \%)$ and abdomen $(6.8 \%)$, genitourinary tract $(3.4 \%)$,cutaneous $(2.7 \%)$ and $4.8 \%$ in others. The category other included 3 patients with disseminated tuberculosis, 1 patient with milliary tuberculosis, and 1 patient with vocal cord tuberculosis, 1 patient with pericarditis and a 1 patient with subclavian pseudo aneurysm.

There are $17 \mathrm{MOH}$ areas in Matara district. The most affected $\mathrm{MOH}$ area was Matara municipal council (11.6\%), followed by Weligama (10.9\%), Malimbada (8.8\%) , Hakmana (8.8\%) , Kamburupitiya (8.8\%) , Akuressa (7.5\%), Devinuwara (6.1\%), Welipitiya (6.1\%) , Dickwella (5.4\%) , Pasgoda (4.8\%) , Thihagoda (4.8\%) , Athuraliya (4.1\%), Kotapola (2.7\%) , Kekanadura (2.7\%) , Morawaka (2.7\%), Mulatiyana (2.7\%) , Kirinda (1.4\%). This $\mathrm{MOH}$ wise distribution is proportionate with the population density of the $\mathrm{MOH}$ areas.

There was only one HIV positive patient in the study group $(0.7 \%)$ which is higher than the national figure which is $<0.1 \%$. There were $9.5 \%$ patientswith diabetics in the study group which is slightly higher than the national figure which is $8.5 \%$. There were no rheumatoid arthritis patients in this population. There were $2.7 \%$ chronic kidney disease patients in the study group which is higher than national prevalence which is $1.3 \%$.There were $1.4 \%$ patients with malignancy which is slightly higher than the national figure which was $1.1 \%$. There were $2 \%$ patients with chronic respiratory diseases which was less than the national figure which was $6 \%$.There were no correlations foundbetween co-morbidities andtreatment outcome.

$79.6 \%$ were non-smokers while $10.9 \%$ had smoked previously and $9.5 \%$ have been smoking currently. $74.8 \%$ of them had not consumed alcohol while $10.9 \%$ had consumed previously and $14.3 \%$ have been consuming alcohol currently. There were no 
patients with other substance abuseamong both genders.There were no correlations found between risk factors and treatment outcome.

144 (98\%) patients has completed anti tuberculosis chemotherapy. This isa very good treatment completion rate as $>90 \%$ treatment success rate is given as the national target. ${ }^{2}$ When compared with the studies conducted by south Asian countries this study showed a high treatment success rate. 6,7 One patient had failed treatment $(0.7 \%)$. Two patients had died due to tuberculosis (1.4\%) which is also less than national figure which lies between 3-5 \%. ${ }^{2}$

\section{Conclusions}

Economically productive age group (15-54) was affected more and there was high prevalence of pleural tuberculosis. The diagnosis of lymphatic tuberculosis is comparatively low. Prevalence of extra pulmonary tuberculosis was high in $\mathrm{MOH}$ areas with high population density which is similar to pulmonary tuberculosis. Prevalence of co-morbidities was higher than the normal population. Significantly high treatment completion rate than the national target indicatesan efficient patient follow-up at District Chest Clinic, Matara

\section{References}

1. EPTB guideline 2013 publication of National Programme for Tuberculosis Control and Chest Diseases Unit, Ministry of Health, Nutrition \& Indigenous Medicine.

2. National manual for tuberculosis control 2016 publication of National Programme for Tuberculosis Control and Chest Diseases Unit, Ministry of Health, Nutrition \& Indigenous Medicine

3. District TB Register 2017 and 2018 -District Chest Clinic, Matara

4. http;//www.statistic.gov.lk Department of census and statistics 2012

5. Ohene SA, Bakker MI, Ojo J, Toonstra A, Awudi D, et al. (2019) Extra-pulmonary tuberculosis: A retrospective study of patients in Accra, Ghana. PLOS ONE 14(1): e0209650.

6. Jamtsho T, Harries AD, The burden and treatment outcomes of extra-pulmonary tuberculosis in Bhutan, Public Health Action 2013 Mar 21; 3(1): 38-42.
7. Ravi Kumar P, Bai PG. A study of extra-pulmonary tuberculosis and its outcome.Int. J Adv. Med. 2017; 4:209--13. 